\title{
Review of: "A previously unrecognized membrane protein in the Rhodobacter sphaeroides LH1-RC photocomplex"
}

Lyudmila Vasilieva

Potential competing interests: The author(s) declared that no potential competing interests exist.

The undoubted and not the only merit of the article is that for the first time a previously unknown protein was discovered in the structure of the RC-LH1 complex of the purple bacterium and its functional role was shown. The high resolution of the complex structure allowed authors to get a lot of new information and draw conclusions about the function of the complex. An important conclusion is drawn from the work of the authors and their colleagues from the UK that the presence of the PufX in Rba. sphaeroides likely correlates with the carotenoid content in LH1 (page 6) and that it is related to quinone transport and phototrophic growth. The extensive additional material in the supplementary increases the valuable content of the work. I fully agree with the authors' own assessment of their work. It really contributes to a deeper understanding of photosynthesis in purple bacteria. 